\title{
Development of a Pressure Sensitive Paint System for Measuring Global Surface Pressures on Rotorcraft Blades
}

\author{
A. Neal Watkins, Bradley D. Leighty, and William E. Lipford \\ Advanced Sensing and Optical Measurement Branch, NASA Langley Research Center, Hampton, VA 23681 \\ Oliver D. Wong \\ US Army, RDECOM, AMRDEC, Aeroflight Dynamics Directorate, Hampton, VA 23681
}

Donald M. Oglesby and JoAnne L. Ingram

Swales Aerospace, Inc., Hampton, VA 23681

\begin{abstract}
This paper will describe the results from a proof of concept test to examine the feasibility of using Pressure Sensitive Paint (PSP) to measure global surface pressures on rotorcraft blades in hover. The test was performed using the U.S. Army 2meter Rotor Test Stand (2MRTS) and 15\% scale swept rotor blades. Data were collected from five blades using both the intensity- and lifetime-based approaches. This paper will also outline several modifications and improvements that are underway to develop a system capable of measuring pressure distributions on up to four blades simultaneously at hover and forward flight conditions.
\end{abstract}

\section{INTRODUCTION}

The accurate determination of spatially continuous pressure and temperature distributions on aerodynamic surfaces is critical for the understanding of complex flow mechanisms and for comparison with computational fluid dynamics (CFD) predictions. Conventional pressure measurements are based on pressure taps and electronically scanned pressure transducers. While these approaches provide accurate pressure information, pressure taps are limited to providing data at discrete points. Moreover, the integration of a sufficient number of pressure taps on a surface can be time and labor intensive and expensive.

This is especially true in rotorcraft research, where the examination of pressure distributions at the rotor tip is vital to advance analytical prediction methods for rotorcraft aerodynamics, acoustics, and interactional effects. There has been considerable research involving pressure measurements on rotor blades [1-4]. However, these measurements typically lack the spatial resolution necessary to capture phenomena such as the nascent tip vortex or dynamic stall. Instrumenting the blades with additional transducers to increase spatial resolution can quickly become prohibitive due to the cost and practicality of fitting a large number of sensors into a small area. In addition, the added centrifugal loads of the pressure transducers can rapidly become unmanageable.

Applying pressure sensitive paint (PSP) to the surface may enable high spatial resolution surface measurements on helicopter rotor blades, thus allowing more accurate analytical prediction methods to be developed. The PSP technique [5-9] exploits the oxygen $\left(\mathrm{O}_{2}\right)$ sensitivity of luminescent probe molecules suspended in gas-permeable binder materials. If the test surface under study is immersed in an atmosphere containing $\mathrm{O}_{2}$ (e.g. air), the recovered luminescence intensity can be described by the Stern-Volmer relationship [10]

$$
\frac{I_{0}}{I}=1+K_{S V} P_{O_{2}}
$$

where $\mathrm{I}_{0}$ is the luminescence intensity in the absence of $\mathrm{O}_{2}$ (i.e. vacuum), I is the luminescence intensity at some partial pressure of oxygen $\mathrm{P}_{\mathrm{O} 2}$, and $\mathrm{K}_{\mathrm{SV}}$ is the Stern-Volmer constant.

Since it is a practical impossibility to measure $\mathrm{I}_{0}$ in a wind tunnel application, a modified form of the Stern-Volmer equation is typically used. This form replaces the vacuum calibration $\left(\mathrm{I}_{0}\right)$ with a reference standard

$$
\frac{I_{R E F}}{I}=A(T)+B(T) \frac{P}{P_{R E F}}
$$

where $I_{R E F}$ is the recovered luminescence intensity at a reference pressure, $\mathrm{P}_{\mathrm{REF}}$.

There are two methods for acquiring PSP data. The most common method used for data acquisition is an "intensitybased" technique. During intensity-based PSP experiments, $I_{R E F}$ is typically acquired while the wind tunnel is off or at very low speed and $P_{\text {REF }}$ is the static pressure when no wind is applied. Thus $\mathrm{I}_{\mathrm{REF}}$ is referred to as the "wind-off" intensity. I is the recovered luminescence intensity at some pressure P. Since this data is collected at a specific condition in the wind tunnel, I is also referred to as the "wind-on" intensity. A(T) and $\mathrm{B}(\mathrm{T})$ are temperature dependent constants for a given PSP formulation and are usually determined before hand using laboratory calibration procedures.

A second method of PSP data acquisition is known as "lifetime-based" PSP [11-15]. In the lifetime-based technique, excitation of the PSP is accomplished using a modulated light source (e.g. laser, flash lamp, or pulsed LED arrays). A fast framing camera (intensified CCD or interline transfer CCD) is used to collect the excited state luminescence decay. Typically 


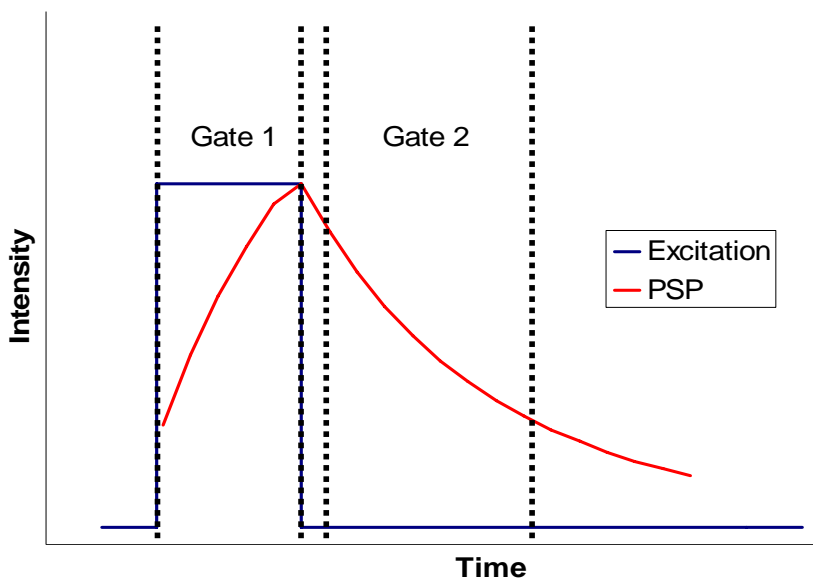

Figure 1. Schematic representation of lifetime-based data acquisition showing excitation (blue) and measured emission (red). The gate regions represent example Gate 1 (during excitation) and Gate 2 (after excitation).

the decay is approximated by acquiring two or more images at different delay times during and/or after the pulsed excitation and integrating photons for fixed periods of time (i.e. gate widths) that have been predetermined to maximize the pressure sensitivity, as demonstrated in Fig. 1. The first image (Gate 1) usually consists of a short gate width and is collected either during the excitation pulse or shortly after it ends. This can be thought of as the reference image because the excited-state decay has the least pressure sensitivity. The second image (Gate 2) is taken at a later time after the excitation pulse and usually has a longer gate width, ensuring maximum pressure (and temperature) sensitivity. More information on the lifetime technique used in this work can be found in [15].

This paper will present results from a proof of concept test for using PSP to measure surface pressure distributions on rotorcraft blades in hover. In addition, it will describe several modifications and improvements to enable the development of a system capable of measuring surface pressure distributions from multiple blades simultaneously in either hover or forward flight conditions.

\section{EXPERIMENTAL}

The U.S. Army 2-meter Rotor Test Stand (2MRTS) was used for this test. The 2MRTS is a general research drive system that allows testing of different fuselage configurations. It is powered by a $47 \mathrm{hp}$ water cooled electric motor driving a 5:1 transmission. A five-bladed fully articulated hub was used. One cuff on the hub was instrumented to measure lead-lag and flapping. The 2MRTS can be fitted with two strain gage force balances: one for rotor loads and one for fuselage loads. The fuselage balance was not monitored since a fuselage was not used for this work. Additional instrumentation included accelerometers for monitoring system health and an encoder to provide $1 / \mathrm{rev}$ and $1024 / \mathrm{rev}$ timing pulses. A more thorough review of the 2MRTS can be found in [16].

The rotor blades used in this test were constructed from carbon fiber, fiberglass, and aromatic nylon fiber honeycomb.
Each blade was painted with a white basecoat to enhance the PSP luminescent output (by reflecting it away from the surface and to the camera). The outer $15 \%$ of each blade was coated with a sol-gel-based PSP formulation using ruthenium bathophenanthroline (Rubath) as the luminophore [15]. This probe was chosen specifically due to its relatively short excited-state lifetime (approximately $5 \mu$ s at vacuum), allowing for an effective freezing of the blade motion mitigating excessive blur as images are acquired. Each blade was marked with 6 fiducial marks to enable registration between the reference and wind-on images, as detailed in Fig. 2. Illumination of the paint was accomplished using $460 \mathrm{~nm}$ light from four LED lamps capable of operating at high repetition rates (up to $5 \mathrm{kHz}$ at pulse widths of $10 \mu \mathrm{s}$ ).

PSP data were collected using both intensity-based and lifetime-based acquisition methods. Timing for both techniques was accomplished using a programmable logic controller and the signals from the $1 / \mathrm{rev}$ and $1024 / \mathrm{rev}$ encoders. This ensured that images were collected from a blade at the same position through multiple revolutions.

For the intensity-based technique, images were acquired using a cooled scientific grade 16-bit resolution CCD camera. The camera chip consisted of $512 \times 512$ pixels, each with a dimension of $24.7 \times 24.7 \mu \mathrm{m}$. A typical wind-on image was collected by opening the mechanical shutter of the camera and flashing the LED arrays for $10 \mu \mathrm{s}$ when the blade was in position. Several hundred revolutions (typically between 100 and 300) of the blade were interrogated for each image to ensure adequate signal-to-noise ratio (SNR). Due to varying thrust angles and blade bending, shims were used to place the blade in the correct hub position to collect wind-off images after each run.

Images for the lifetime-based technique were collected using an interline CCD camera containing a hardware accumulator. The camera operates by masking every other line of the chip, allowing for charge to be transferred quickly ( $\sim 200 \mathrm{~ns}$ transfer time) from the unmasked region to the masked region for either storage or readout. The active area of the CCD chip is $1392 \times 1040$ pixels $(6.45 \times 6.45 \mu \mathrm{m})$ and the digitizer operates at 12-bit resolution. The camera is equipped with a fast electronic shutter capable of acquiring images as fast as $1 \mu \mathrm{s}$. The hardware accumulator allows for multiple light pulses to

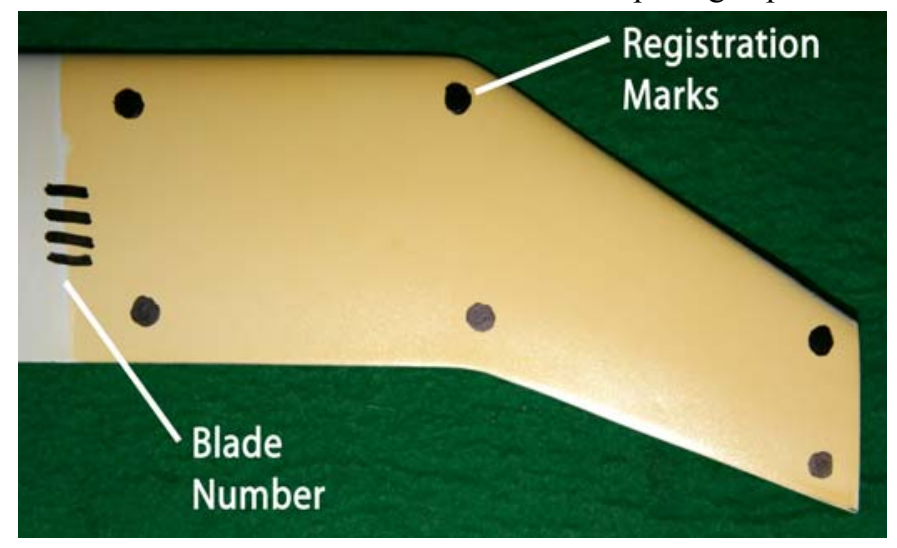

Figure 2. Painted blade tip showing fiducial (registration) marks and blade number markings. 


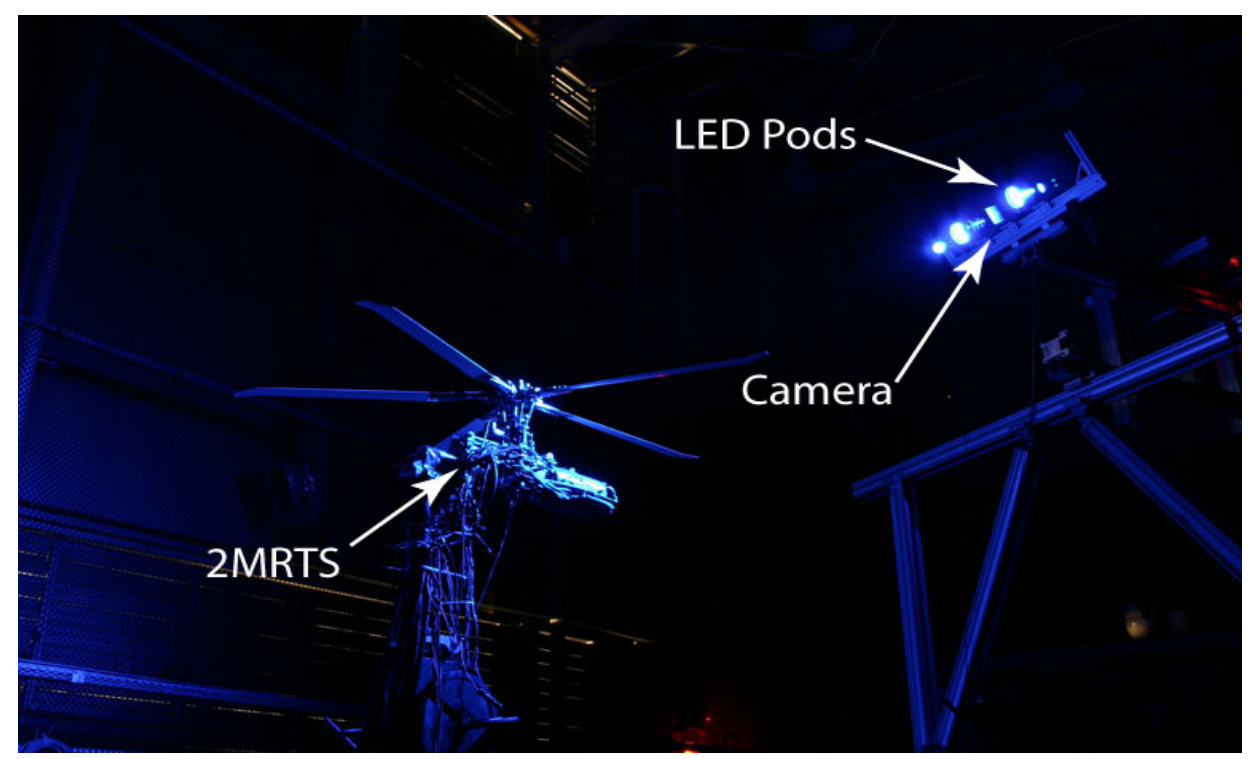

Figure 3. 2MRTS, PSP camera, lights and structure in the Rotor Test Cell

be added together, achieving the required SNR needed for lowlight techniques without the added noise attributed to standard intensifiers. For this test, images were again acquired using a $10 \mu \mathrm{s}$ LED flash. The first image (Gate 1) was collected from 5-10 $\mu$ s (during the pulse) and the second image (Gate 2) was collected from $15-35 \mu \mathrm{s}$ ( $5 \mu \mathrm{s}$ after the excitation pulse had ended). To increase collection efficiency, the CCD chip was binned horizontally and vertically by 4 (effective imaging area of $348 \times 260$ pixels).

All testing was conducted in the Rotor Test Cell (RTC) at the NASA Langley Research Center 14x22-Foot Subsonic Tunnel. The RTC is an open room used for propeller static testing and rotor hover testing. Louvers at the bottom and top of the walls are opened to minimize recirculation. The 2MRTS is mounted in the east end of the RTC to a blade strut that places the rotor $2.83 \mathrm{~m}(9.3 \mathrm{ft})$ above the floor. PSP lights and cameras were mounted on an aluminum structure that was placed so that its closest member was $2.4 \mathrm{~m}(7.9 \mathrm{ft}$ or 2.67 rotor radii) from the rotor, as shown in Fig. 3. The camera and lights were mounted onto an extension of the frame so that the distance between the camera/lights and the rotor could be varied both horizontally $\left(\mathrm{S}_{\mathrm{x}}\right)$ and vertically $\left(\mathrm{S}_{\mathrm{z}}\right)$ as shown in Fig. 4. PSP data were collected using two viewing angles, as depicted in Fig. 5. For the first viewing angle, the camera and lights were located outboard $\left(\mathrm{S}_{\mathrm{x}}\right)$ of the rotor 1.35 radii $(1.22 \mathrm{~m}$ or $4 \mathrm{ft})$ and above the rotor tip $\left(\mathrm{S}_{\mathrm{z}}\right)$ by 0.76 radii $(0.70 \mathrm{~m}$ or 2.3 $\mathrm{ft})$. The separation distances are measured from the point where the blade passes closest to the camera/lights. The second viewing angle was chosen to collect data from a different azimuthal angle with the vertical separation increased to 1.35 radii, equal to the horizontal separation. A more thorough description of the mounting and measurement regions as well as test conditions is provided in [17].
PSP results were obtained during collective sweeps of the rotor where the angle of attack for each blade was changed simultaneously. The sweeps ranged from $6^{\circ}$ to $12^{\circ}$ in increments of $2^{\circ}$ and the measured thrust at each condition was used to determine the thrust coefficient $\left(\mathrm{C}_{\mathrm{T}}\right)$ according to

$$
C_{T}=\frac{T}{\pi R^{2} \rho(\Omega R)^{2}}
$$

where $T$ is the measured thrust, $R$ is the blade radius, $\rho$ is the air density, and $\Omega$ is the rotation speed. The thrust coefficients for each collective angle are listed in Table 1.

\section{RESUlts AND Discussion}

\section{A. Intensity vs. Lifetime Techniques}

Results from the intensity-based acquisition method suffered from excessive poor registration between the wind-on and wind-off images, as seen in Fig. 6. Even though the blade cuff was positioned identically between the wind-off and wind-on images, blade flex between the cuff and the tip resulted in the blade tip being in a significantly different position under load. This causes the resulting ratioed image to have significant areas that are immeasurable, especially around the edges and registration marks.

The images collected using the lifetime-based technique did

TABLE 1

Thrust Coefficient for EAch Collective Angle

\begin{tabular}{|c|c|}
\hline $\begin{array}{c}\text { Collective } \\
\text { (deg) }\end{array}$ & $\begin{array}{c}\text { Thrust } \\
\text { Coefficient }\end{array}$ \\
\hline 6 & 0.004 \\
\hline 8 & 0.005 \\
\hline 10 & 0.007 \\
\hline 12 & 0.009 \\
\hline
\end{tabular}




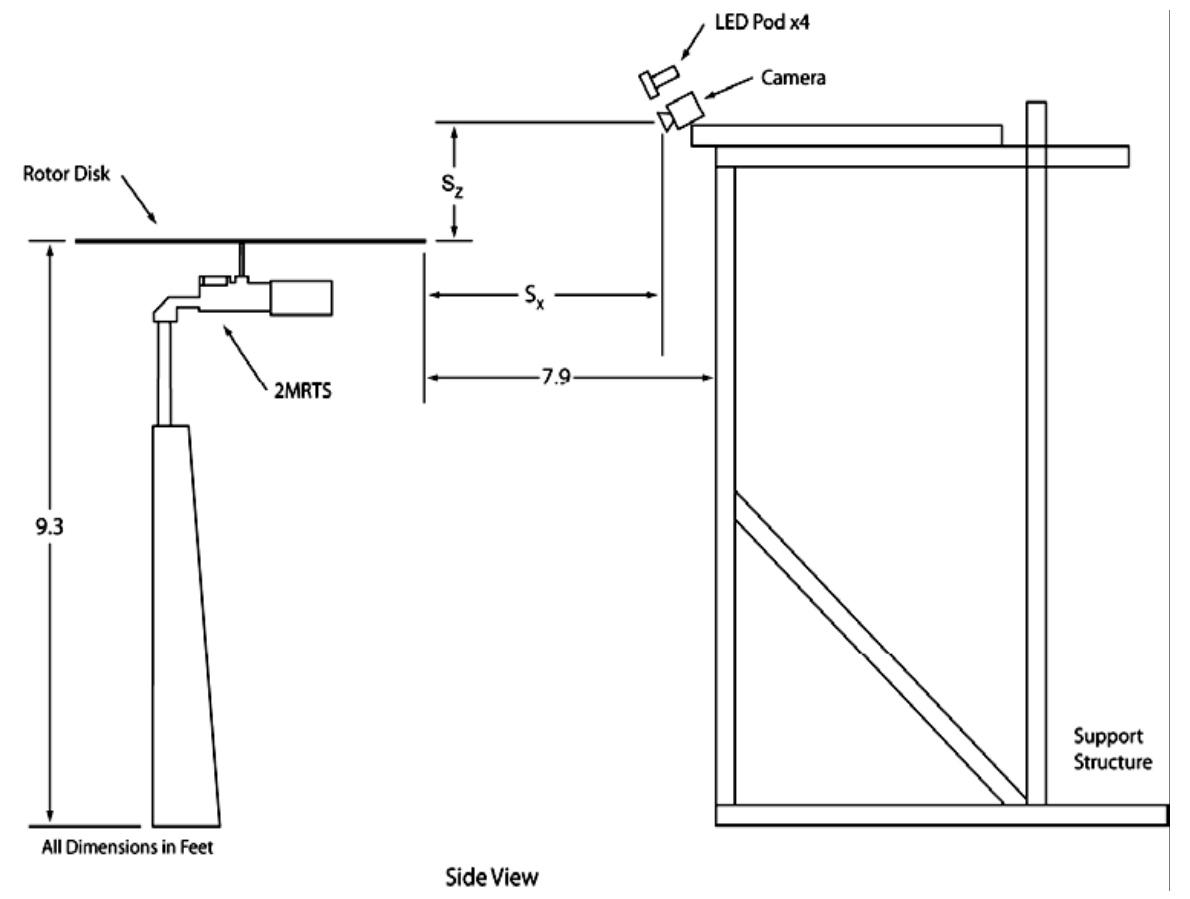

Figure 4. Experimental setup showing the mounting frame for the camera and lights. $\mathrm{S}_{\mathrm{x}}$ and $\mathrm{S}_{\mathrm{y}}$ are the horizontal and vertical separation between the camera and the closest approach of the blade, respectively.

not suffer from these registration errors as all images were collected at condition, thus with the blade tip under the same load. However, some blurriness of the lifetime images was observed, as seen in Fig. 7. This blurriness was a result of slight variations in blade position due to flapping, leading, and lagging of the blade over the several hundred flashes (revolutions) required for data acquisition. The registration errors between the Gate 1 and Gate 2 images, however, were significantly reduced when compared to the intensity-based method, as shown in Fig. 6. As a result, only data collected using the lifetime-based technique will be presented.

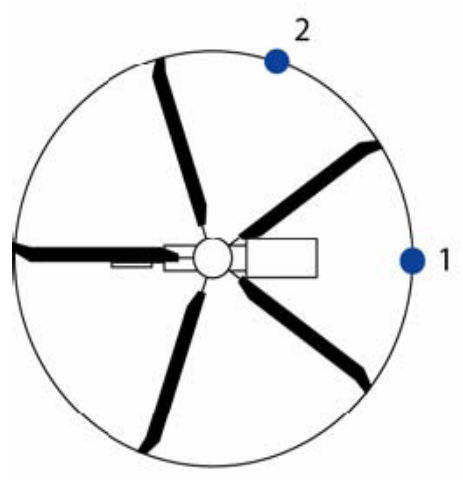

\section{B. Determining Surface Pressure}

Quantitative surface pressures on the rotor blades could not be accurately determined from the recovered PSP data because of the inherent temperature sensitivity of the paint. From calibration data obtained prior to the test, the intensity of the paint decreases by approximately $1.5 \%$ per degree increase in temperature $\left({ }^{\circ} \mathrm{C}\right)$, which corresponds to an apparent pressure change of approximately $2 \mathrm{kPa}(0.3 \mathrm{psi})$. To account for this, independent measurement of the surface temperature or comparison with independent surface pressure measurements (i.e. pressure taps) are required to determine global surface pressure. These measurements are not available owing to the size of the blades. However, because the blade tip speed is

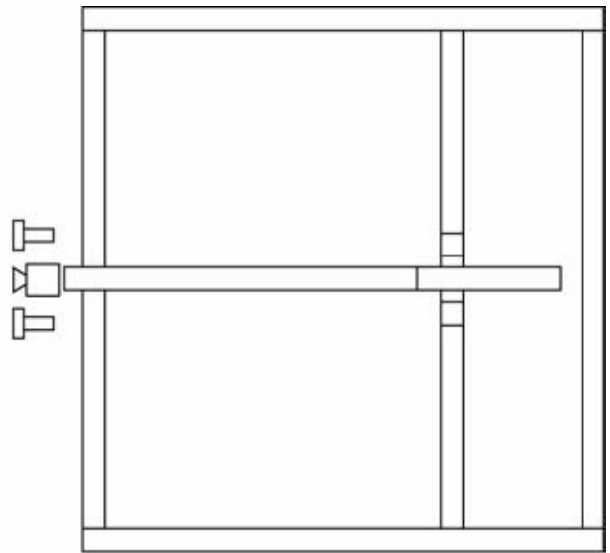




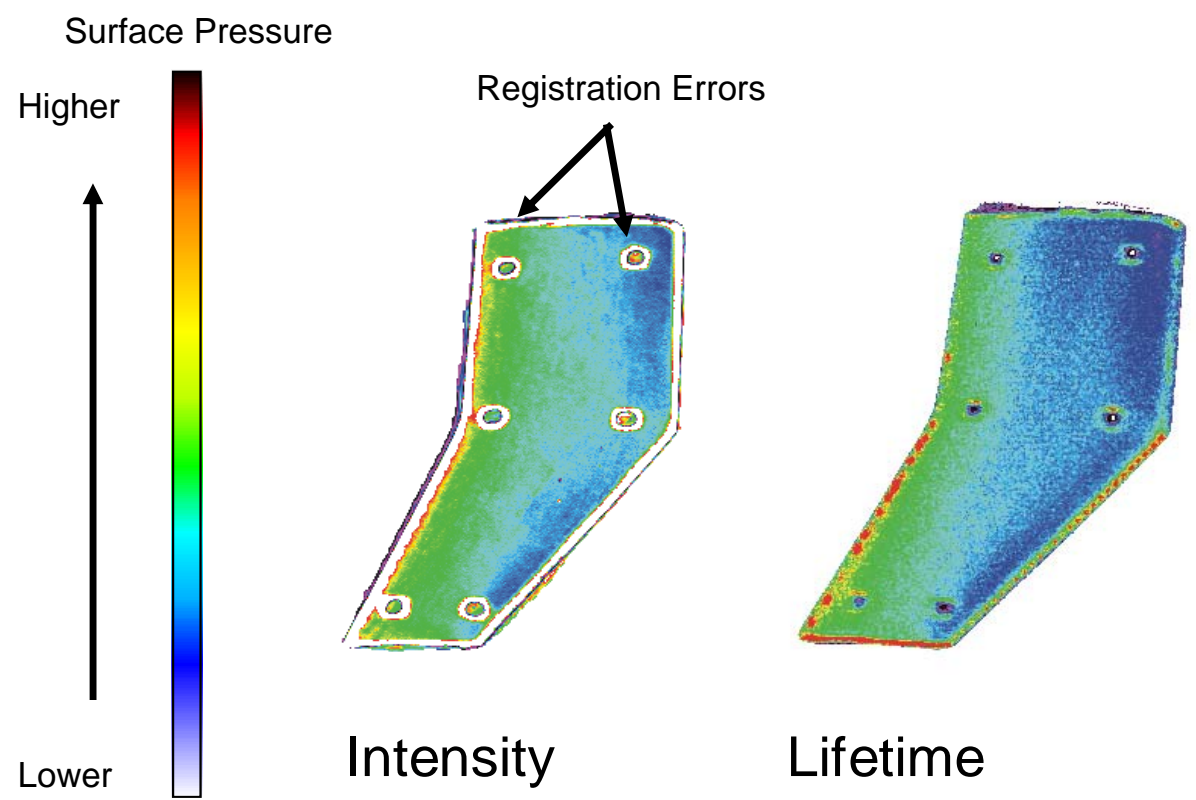

Figure 6. Comparison of surface pressure distributions recovered using intensity-based (left) and lifetime-based (right) acquisition. The model edges and registration marks of the intensity-based acquisition show large discrepancies due to blade tip bending between windoff and wind-on images. These images were acquired from the first viewing angle (see Fig. 5).

subsonic $(221 \mathrm{~m} / \mathrm{s})$, temperature gradient across the blade are assumed to be minimal, thus allowing at least a qualitative analysis of pressure distributions using PSP.

\section{Lifetime Results}

Fig. 8 shows the measured surface pressure distributions across blade 1 at thrust coefficients ranging from 0.004 to 0.009. At the lowest thrust condition $\left(\mathrm{C}_{\mathrm{T}}=0.004\right)$ the pressure distribution shows a slight lower pressure region near the leading edge of the blade, which gradually increases towards the trailing edge. As the thrust increases, this low pressure region becomes better defined until at the highest thrust

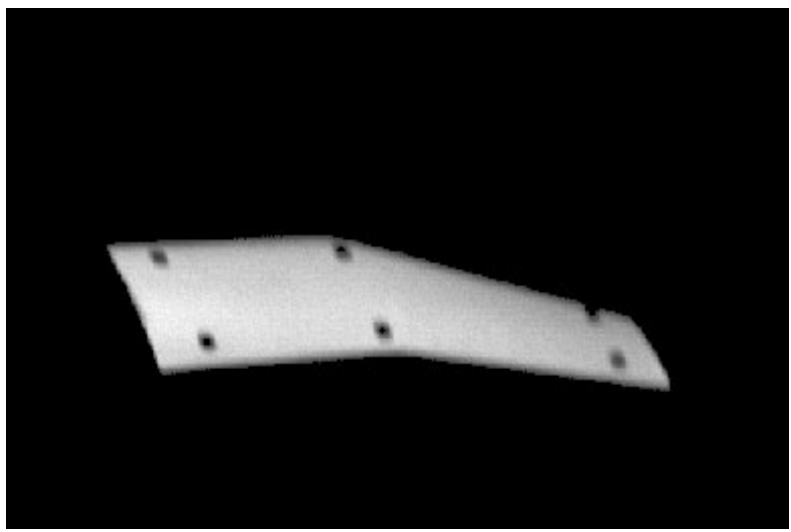

Figure 7. Typical raw lifetime image $\left(\mathrm{C}_{\mathrm{T}}=0.004\right)$ showing blur due to flapping, leading and lagging during data acquisition. condition $\left(\mathrm{C}_{\mathrm{T}}=0.009\right)$, the pressure distribution has a very strong and well defined low pressure region. In addition, it appears to turn the corner and exist for a short chord-wise distance at the tip.

It should be noted that these are two-dimensional images of the blade tip and due to observation location, collective, blade twist, flapping and leading-edge angles, etc., the entire upper surface is not visible at all conditions. This changes across all thrust conditions as is evidenced by the different positions of the registration marks. In general, at the higher thrust conditions, the blade twists such that more of the leading edge is visible to the camera. The registration marks at the leading edge are about $6.35 \mathrm{~mm}$ (0.25 in) from the leading edge, which corresponds to an approximate $\mathrm{x} / \mathrm{c}$ of 0.1 . As a result of these effects, there is a fair degree of uncertainty in how much data is available forward of $\mathrm{x} / \mathrm{c} \approx 0.1$, especially at the lower thrust conditions.

The pressure distributions on all five blades at $\mathrm{C}_{\mathrm{T}}=0.009$ are shown in Fig. 9. Blades 1, 3, 4, and 5 show similar qualitative responses with minor variations in the strength of the suction peak, most likely due to slight manufacturing differences in the blades themselves. However, blade 2 displays a significant deviation, with the suction peak almost nonexistent. This was evident in all of the thrust conditions. Blade 2 was the most difficult to track and cursory inspection of the paint showed no obvious differences with the other four blades. It is speculated that this is a maverick blade (a blade that behaves differently from the other blades, generally due to manufacturing differences), but without additional pressure instrumentation, this cannot definitely be determined. 


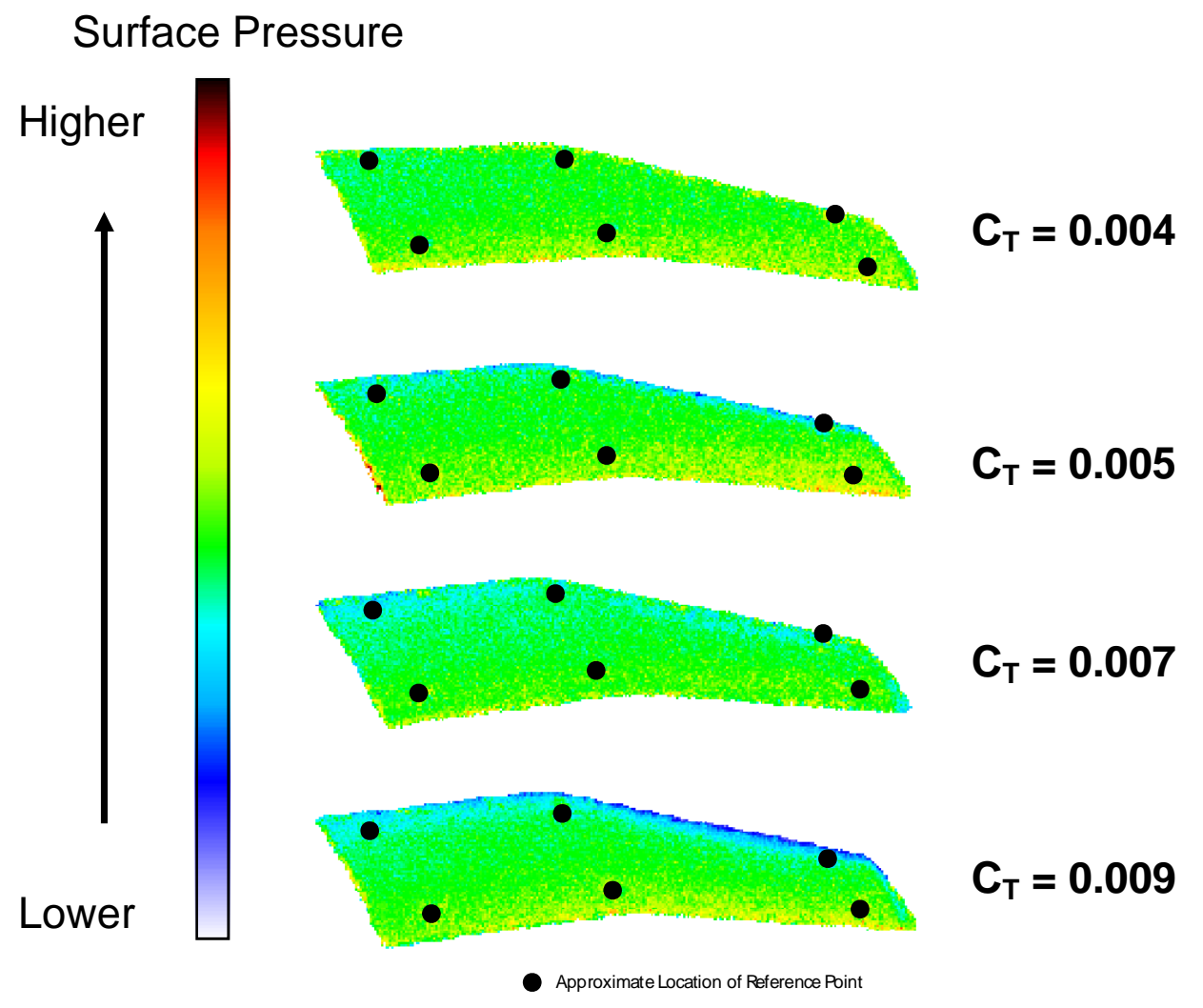

Figure 8. Recovered global surface pressure distributions from a rotorcraft blade (blade 1) in hover with increasing thrust angle $\left(\mathrm{C}_{\mathrm{T}}\right)$.

\section{FUTURE DIRECTIONS}

The preceding results show that it is feasible to use PSP to measure global surface pressure distributions on rotors. However, due to instrumentation limitations, the results suffer from excessive noise. In addition, without additional pressure instrumentation, there is no means to completely validate the technique. Several improvements to the system are currently underway to address these issues for a second proof of concept test that will occur in the upcoming months.

First, new LED arrays have been acquired that use superbright $400 \mathrm{~nm}$ LEDs to provide illumination. The $400 \mathrm{~nm}$ illumination will excite both of the most common luminophores used in PSP (Rubath and platinum mesotetra(pentafluorophenyl) porphine $[\mathrm{Pt}(\mathrm{TfPP})])$. The array has been designed to operate in either continuous mode (always on), pulse on demand (LED turns on with TTL pulse), or overdriven for short pulse operation (up to $5 \mathrm{kHz}$ ). The individual LEDs are surface mounted with a cone angle of $160^{\circ}$. This yields an extremely uniform, wide illumination field that provides approximately 5 times more light than the previous LED arrays. To increase the light output even more, focusing attachments utilizing $152.4 \mathrm{~mm}$ (6 in) Fresnel lenses have been designed, and are shown in Fig. 10. These attachments work to focus the light for use in distances up to approximately $7.6 \mathrm{~m}(25 \mathrm{ft})$ and provide an order of magnitude improvement in illumination field intensity. Utilizing several of these LED arrays with focusing attachments should increase illumination intensity by a factor of 100 or more, allowing for greater spatial resolution images to be acquired (less binning) and reducing overall data acquisition time (lower number of pulses needed to fill the electronic well of the CCD).

Second, higher spatial and digital resolution cameras have been developed for use with the lifetime technique. These cameras employ a CCD chip with an active area of $2048 \times 2048$ pixels (pixel area is $7.4 \times 7.4 \mu \mathrm{m}$ ) and essentially the same peak quantum efficiency. It employs 14-bit digitization for up to four times better digital resolution, and also has on-board memory that will allow it to rapidly store images in the camera, making it possible to run multiple cameras simultaneously from the same computer platform.

Finally, an experiment has been designed to validate the onblade PSP measurements. This experiment uses the larger General Rotor Model System (GRMS) rotor test stand. The larger blades on the GRMS provide more room to embed dynamic pressure transducers in the blade. These blades are constant chord with a swept-tapered tip and a 14 degree linear twist distribution. They use the RC family of airfoils. The upper portion of Fig. 11 shows the distribution of airfoils and dimensions of the blades. Of the four blades, two are pressure instrumented. The first instrumented blade has two rows of chord-wise dynamic pressure transducers, with the rows located at the $93 \%$ and $99 \%$ radial stations. The second blade 


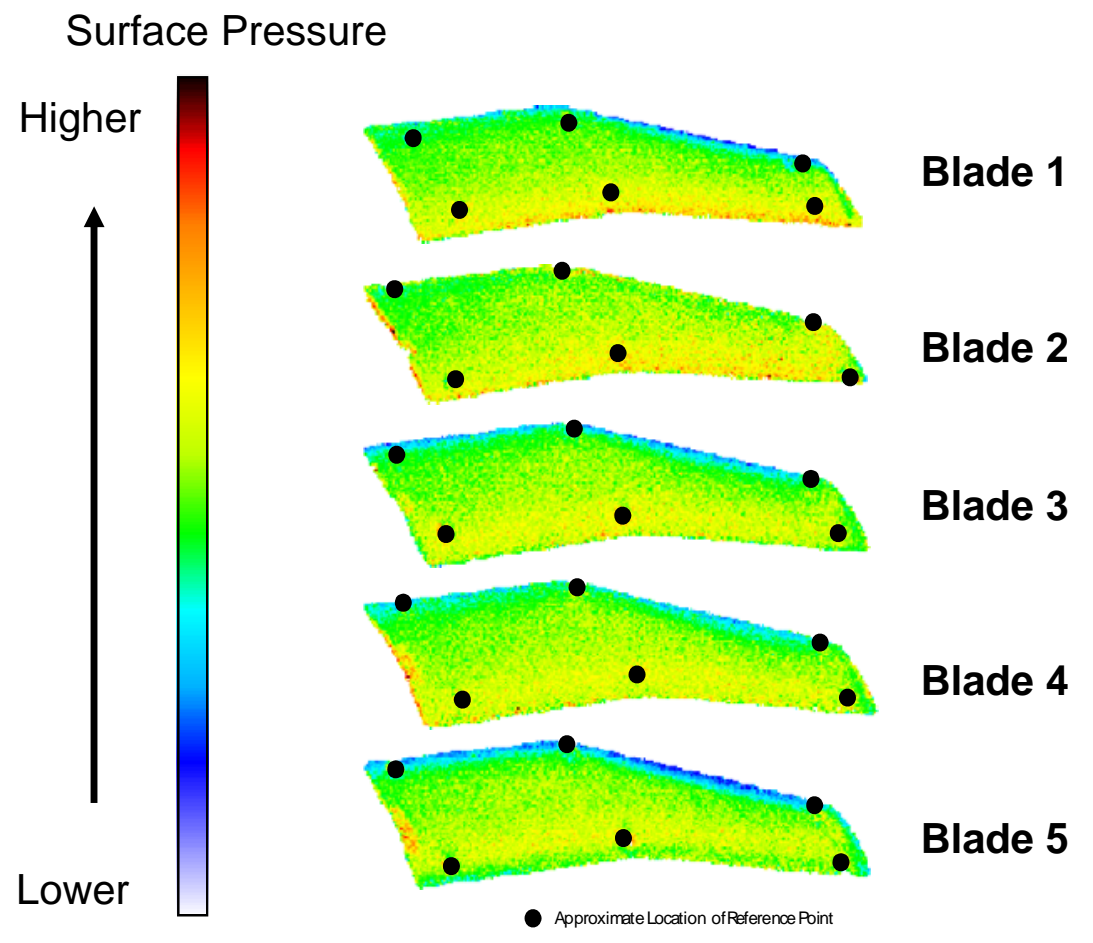

Figure 9. Blade-to-blade variation at $\mathrm{C}_{\mathrm{T}}=0.009$. The small artifacts at the inboard edge are due to marks placed for blade numbering.

has one chord-wise row at $93 \%$ radius. Each row has 10 pressure transducers located on the upper surface, as shown in the lower portion of Fig. 11.

\section{CONCLUSION}

The results from a proof of concept test using PSP to measure surface distributions on rotor blades at hover condition have been presented. These tests were conducted on a set of 5 blades at moderate thrust conditions. Both intensitybased and lifetime-based acquisition methods were employed and it was determined that due to the large amount of blade

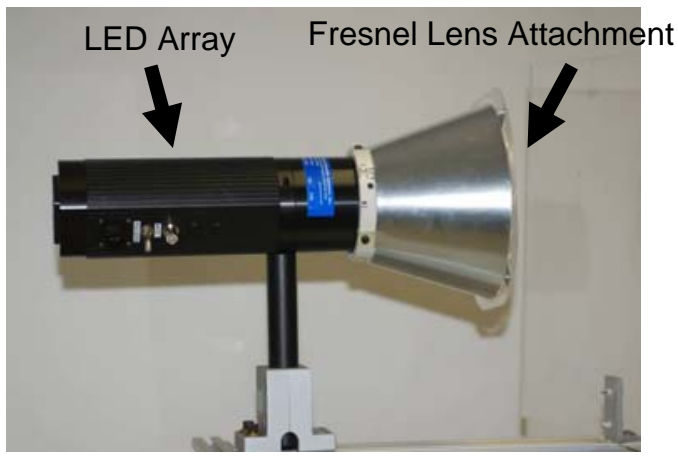

Figure 10. Improved LED-based array including Fresnel lens attachment for focusing the illumination field at distances greater than $\sim 2.5 \mathrm{~m}$. flexing during rotor operation, the lifetime-based method must be used because all images are acquired at condition. Qualitatively, the pressure distributions across the surface of the blades agree with expected results, but further validation of the technique is still required.

Several modifications and improvements to the system have been identified. More and brighter illumination sources will be used to increase the illumination field, allowing for larger spatial resolution images to be acquired using fewer pulses. Also, new cameras with larger active areas and greater digital resolution have been acquired and are currently being tested. Finally, a new set of blades to include instrumented blades have been designed and fabricated for use in calibrating the PSP and for validation.

\section{ACKNOWLEDGMENTS}

The authors would like to acknowledge W. Derry Mace, Curtis Fossum, and Andrew Harrison for their assistance during this test.

\section{REFERENCES}

[1] P.F. Lorber, R.C. Stauter, and A.J. Landgrebe, "A Comprehensive Hover Test of the Airloads and Airflow of an Extensively Instrumented Model Helicopter Rotor," in Proc. 45th Ann. Forum American Helicopter Society, Boston, MA., May 1989. 


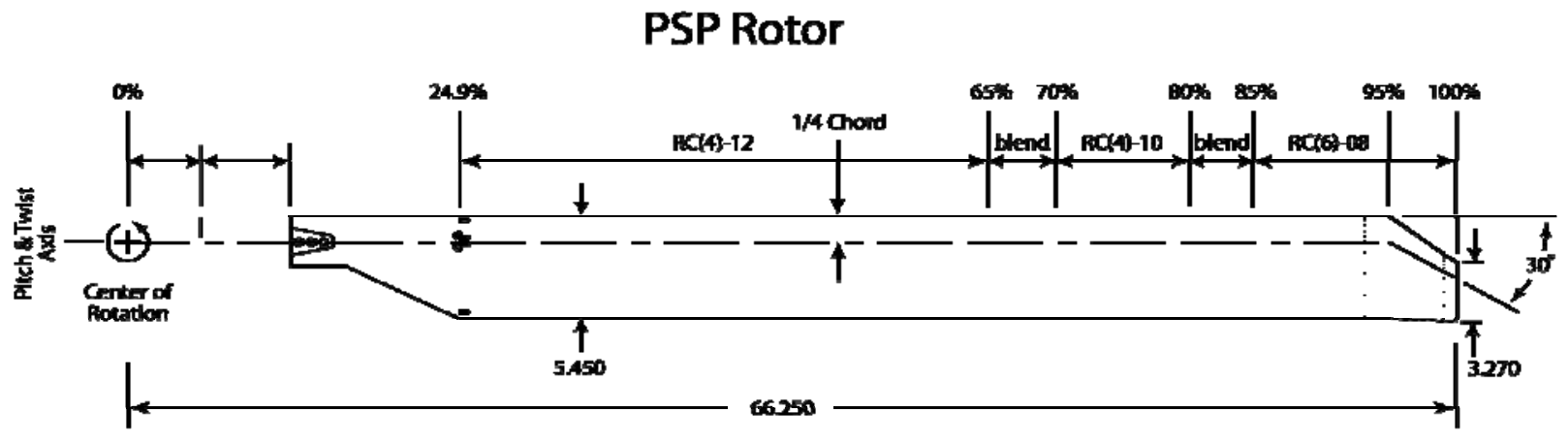

PSP Rotor Instrumentation Locations

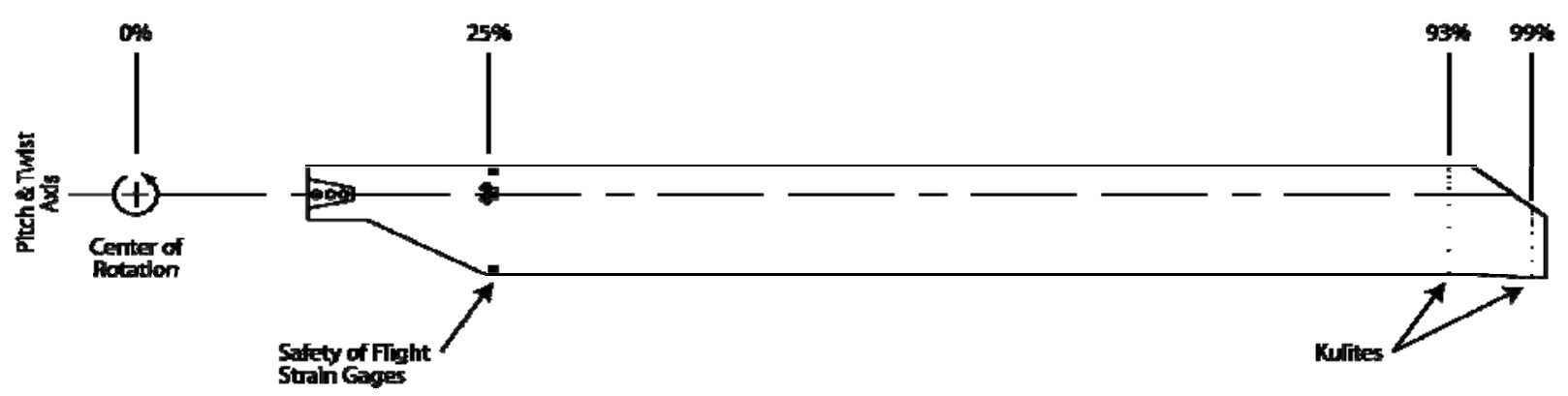

Figure 11. Pressure sensitive paint General Model Rotor System rotor blade. The upper diagram shows the distribution of the airfoils and dimensions of the blades. The lower diagram shows the rotor instrumentation locations.

[2] M.K. Lal, S.G. Liou, G.A. Pierce, and N.M. Komerath, N.M., "Measurements around a Rotor Blade Excited In Pitch, Part 2: Unsteady Surface Pressure," Journal of the American Helicopter Society, vol. 39, no. 2, pp. 13 - 20, April 1994.

[3] S.A. Gorton, D.R. Poling, and L. Dadone, "Laser Velocimetry and Blade Pressure Measurements of a Blade-Vortex Interaction," Journal of the American Helicopter Society, vol. 40, no. 2, pp. 15-23, April 1995.

[4] P.F. Lorber, "Aerodynamic Results of a PressureInstrumented Model Rotor Test at the DNW", in Proc. 46th Ann. Forum American Helicopter Society, Washington, DC., May 1990.

[5] Kavandi, J. et al., "Luminescence Barometry in Wind Tunnels," Rev. Sci. Instrum., vol. 61, no. 11, pp. 33403347, Nov. 1990.

[6] M.J. Morris, M.E. Benne, R.C. Crites, and J.F. Donovan, "Aerodynamic Measurements Based in Photoluminescence," presented at the $31^{\text {st }}$ Aerospace Sciences Meeting and Exhibit, Reno, NV, Jan. 11-14, 1993, Paper 93-0175.

[7] B. McLachlan and J. Bell, "Pressure-Sensitive Paint in Aerodynamic Testing," Exp. Therm. Fluid Sci., vol. 10, no. 4, pp. 470-485, May 1995.

[8] T. Liu, B. Campbell, S. Burns, and J. Sullivan, "Temperature- and Pressure-Sensitive Luminescent Paints in Aerodynamics," Appl. Mech. Rev., vol. 50, no. 4, pp. 227-246, 1997.
[9] T. Liu and J.P. Sullivan, Pressure and Temperature Sensitive Paints (Experimental Fluid Dynamics), Berlin: Springer-Verlag, 2004.

[10] J. Lakowicz,, Principles of Fluorescence Spectroscopy, Second Edition, New York: Kluwer Academic/Plenum Publishers, 1999, pp. 239-242.

[11] R. Engler and C. Klein, "DLR PSP System: Intensity and Lifetime Measurements," in Proc. $17^{\text {th }}$ Int. Congr. Instrumentation in Aerospace Simulation Facilities, Pacific Grove, CA, 1997, pp. 46-56.

[12] J. Holmes, "Analysis of Radiometric, Lifetime and Fluorescent Imaging for Pressure Sensitive Paint," Aeronaut. J., vol. 102, no. 1014, pp. 189-194, 1998.

[13] J.H. Bell, T.E. Schairer, L.A. Hand, and R.D. Mehta, "Surface Pressure Measurements using Luminescent Coatings," Annu. Rev. Fluid Mech., vol. 33, pp. 115-206, Jan. 2001.

[14] K. Mitsuo, Y. Egami, K. Asai, H. Suzuki, and H. Mizushima, 2002, "Development of Lifetime Imaging System for Pressure-Sensitive Paint," presented at the $22^{\text {nd }}$ AIAA Aerodynamic Measurement Technology and Ground Testing Conference, St. Louis, MO, June 24-26, 2002, Paper 2002-2909.

[15] A.N. Watkins, J.D. Jordan, B.D. Leighty, J.L. Ingram, and D.M. Oglesby, "Development of Next Generation Lifetime PSP Imaging Systems," Proc. 20th Int. Congr. Instrumentation in Aerospace Facilities, Gottingen, Germany, 2003, pp. 372-382. 
[16] A.E. Phelps and J.D. Berry, "Description of the U.S. Army Small-Scale 2-Meter Rotor Test System", NASA Langley Research Center, Hampton, VA, TM-87762, AVSCOM TM 86-B-4, 1987.
[17] O.D. Wong, A.N. Watkins, and J.L. Ingram, "Pressure Sensitive Paint Measurements on $15 \%$ Scale Rotor Blades in Hover", presented at the $35^{\text {th }}$ AIAA Fluid Dynamics Conference and Exhibit, Toronto, Ontario, Canada, June 6-9, 2005, Paper 2005-5008. 